\title{
A GENERAL COINCIDENCE AND FIXED POINT THEOREM FOR HYBRID PAIRS OF MAPPINGS SATISFYING A COMMON COINCIDENCE RANGE PROPERTY
}

\begin{abstract}
In this paper the notion of common coincidence range property is introduced and a general coincidence and fixed point theorem is proved. As applications, new results for mappings satisfying a contractive condition of integral type and for mappings satisfying a $\phi$-contractive condition are obtained.
\end{abstract}

KEY WORDS: coincidence point, fixed point, hybrid pairs, common coincidence range property, implicit relation, weakly altering distance.

AMS Mathematics Subject Classification: 54H25, 47H10.

\section{Introduction and preliminaries}

Let $(X, d)$ be a metric space. We denote by $C B(X)$ the set of all nonempty closed bounded subsets of $(X, d)$ and by $H$ the Hausdorff-Pompeiu metric on $X$, i.e.

$$
H(A, B)=\max \left\{\sup _{x \in A}\left\{d(x, B), \sup _{y \in B} d(y, A)\right\},\right.
$$

where $A, B \in C B(X)$ and

$$
d(x, A)=\inf _{y \in A}\{d(x, y)\}
$$

Definition 1. Let $f: X \rightarrow X$ and $F: X \rightarrow 2^{X}$ be.

1) A point $x \in X$ is said to be a coincidence point of $f$ and $F$ if $f x \in F x$.

The set of all coincidence points of $f$ and $F$ is denoted by $\mathcal{C}(f, F)$.

2) A point $x \in X$ is a common fixed point of $f$ and $F$ if $x=f x \in F x$.

3) The pair of functions $f$ and $F$ is denoted by $(f, F)$ and is named a hybrid pair of mappings. 
Definition 2. Let $(f, F)$ be a hybrid pair of mappings.

1) The mapping $f: X \rightarrow X$ is said to be coincidentally idempotent with respect to $F[3]$ if $x \in \mathcal{C}(f, F)$ implies $f x=f f x$.

2) The mapping $f: X \rightarrow X$ is said to be occasionally coincidentally idempotent with respect to $F[8]$ if there exists $u \in \mathcal{C}(f, F)$ such that $f u=$ $f f u$.

In 2011, Sintunavarat and Kumam [20] introduced the notion of common limit range property for a pair of two single valued mappings. Imdad et al. [4] introduced the notion of common limit range property for a hybrid pair of mappings.

Quite recently, Imdad et al. [5] introduced the notion of joint common limit range property for two pairs of hybrid mappings. Another form of joint common limit range property for hybrid pairs of mappings is introduced in [1].

Definition $3([5])$. Let $f, g: X \rightarrow X$ be two single valued mappings and $F, G: X \rightarrow C B(X)$ be two multivalued mappings of a metric space $(X, d)$. The pairs of mappings $(f, F)$ and $(g, G)$ are said to have joint common limit range property, denoted (JCLR) - property, if there exist two sequences $\left\{x_{n}\right\}$ and $\left\{y_{n}\right\}$ in $X$ and $A, B \in C B(X)$ such that

$$
\begin{gathered}
\lim _{n \rightarrow \infty} F x_{n}=A, \quad \lim _{n \rightarrow \infty} G y_{n}=B, \\
\lim _{n \rightarrow \infty} f x_{n}=\lim _{n \rightarrow \infty} g y_{n}=t \in A \cap B \cap f(X) \cap g(X) .
\end{gathered}
$$

We introduce the notion of common coincidence range property without the limit of sequences.

Definition 4. Let $(X, d)$ be a metric space, $S, T: X \rightarrow X$ and $A: X \rightarrow$ $2^{X}$. The pair $(A, S)$ is said to have a common coincidence range property with respect to $T$, denoted $C P_{(A, S) T}$, if there exists $z=S x \in A x$ for some $x \in X$ with $z \in T(X)$.

Example 1. Let $X=[0, \infty)$ with usual metric and $S x=\frac{x^{2}+1}{2}, T x=$ $x+\frac{1}{4}$ and $A x=\left[\frac{1}{4}, 1\right]$. Then $S 0=\frac{1}{2} \in A 0=\left[\frac{1}{4}, 1\right]$. Hence, there exists $z=\frac{1}{2} \in T(X)=\left[\frac{1}{4}, \infty\right)$.

Remark 1. It is obvious that the Definition 4 is not using the limit of sequences from $(X, d)$.

Definition $5([6])$. An altering distance is a mapping $\psi:[0, \infty) \rightarrow[0, \infty)$ which satisfies 
$\left(\psi_{1}\right): \psi$ is increasing and continuous,

$\left(\psi_{2}\right): \psi(t)=0$ if and only if $t=0$.

Fixed point problems involving altering distances have been studied in [16], [18], [19] and in other papers.

Definition 6 ([14]). A weak altering distance is a mapping $\psi:[0, \infty) \rightarrow$ $[0, \infty)$ which satisfies

$\left(\psi_{1}\right): \psi$ is increasing,

$\left(\psi_{2}\right): \psi(t)=0$ if and only if $t=0$.

Example 2. The function $\psi$ defined by $\psi(t)=\left\{\begin{array}{c}t, \quad t \in[0,1] \\ e^{t}, t \in(1, \infty) .\end{array}\right.$ is a weakly altering distance but it is not an altering distance.

The purpose of this paper is to prove a general coincidence and fixed point theorem for two hybrid pairs of mappings $(A, S)$ and $(B, T)$ satisfying an implicit relation without any form of compatibility and without limits of any sequence of points of $(X, d)$, using $C P_{(A, S) T}$-property.

\section{Implicit relations}

Several fixed point theorems and common fixed theorems have been unified considering a general condition by an implicit relation in [9], [10] and in other papers. The study of fixed points for hybrid pairs of mappings satisfying implicit relations is initiated in [11] - [13] and in other papers.

Let $\mathcal{F}_{c}$ be the set of all continuous functions $F: \mathbb{R}_{+}^{6} \rightarrow \mathbb{R}$ satisfying the following conditions [15]:

$\left(F_{1}\right): F$ is nondecreasing in variable $t_{1}$,

$\left(F_{2}\right): F(t, 0,0, t, t, 0)>0, \forall t>0$

$\left(F_{3}\right): F(t, 0, t, 0,0, t)>0, \forall t>0$.

Example 3. $F\left(t_{1}, \ldots, t_{6}\right)=t_{1}-k \max \left\{t_{2}, t_{3}, \ldots, t_{6}\right\}$, where $k \in[0,1)$.

Example 4. $F\left(t_{1}, \ldots, t_{6}\right)=t_{1}-k \max \left\{t_{2}, t_{3}, t_{4}, \frac{t_{5}+t_{6}}{2}\right\}$, where $k \in$ $[0,1)$.

Example 5. $F\left(t_{1}, \ldots, t_{6}\right)=t_{1}-k \max \left\{t_{2}, \frac{k}{2}\left(t_{3}+t_{4}\right), \frac{k}{2}\left(t_{5}+t_{6}\right)\right\}$, where $k \in[0,2)$.

Example 6. $F\left(t_{1}, \ldots, t_{6}\right)=t_{1}-k \max \left\{t_{2}, k\left(t_{3}+t_{4}\right), k\left(t_{5}+t_{6}\right)\right\}$, where $k \in[0,1)$.

Example 7. $F\left(t_{1}, \ldots, t_{6}\right)=t_{1}-a t_{2}-b\left(t_{3}+t_{4}\right)-c \max \left\{t_{3}+t_{4}, t_{5}+t_{6}\right\}$, where $a, b, c \geq 0$ and $a+b+c<1$. 
Example 8. $F\left(t_{1}, \ldots, t_{6}\right)=t_{1}^{2}-c_{1} \max \left\{t_{2}^{2}, t_{3}^{2}, t_{4}^{2}\right\}-c_{2} \max \left\{t_{3} t_{5}, t_{4} t_{6}\right\}-$ $c_{3} t_{5} t_{6}$, where $c_{1}, c_{2}, c_{3} \geq 0$ and $c_{1}<1$.

Example 9. $F\left(t_{1}, \ldots, t_{6}\right)=t_{1}-a t_{2}-b t_{3}-c t_{4}-d t_{5}-e t_{6}$, where $a, b, c, d, e \geq 0$ and $c+d<1, b+e<1$.

Example 10. $F\left(t_{1}, \ldots, t_{6}\right)=t_{1}-\alpha \max \left\{t_{2}, t_{3}, t_{4}\right\}-(1-\alpha)\left(a t_{5}+b t_{6}\right)$, where $\alpha \in(0,1), a, b \geq 0$ and $\max \{a, b\}<1$.

Example 11. $F\left(t_{1}, \ldots, t_{6}\right)=t_{1}-a t_{2}-\frac{b\left(t_{5}+t_{6}\right)}{1+t_{3}+t_{4}}$, where $a, b \geq 0$ and $a+2 b<1$.

Example 12. $F\left(t_{1}, \ldots, t_{6}\right)=t_{1}-\max \left\{c t_{2}, c t_{3}, c t_{4}, a t_{5}+b t_{6}\right\}$, where $c \in$ $(0,1), a, b \geq 0$ and $\max \{a, b\}<1$.

\section{Main results}

Theorem 1. Let $(X, d)$ be a metric space, $A, B: X \rightarrow C B(X)$ and $S, T: X \rightarrow X$ such that for all $x, y \in X$

$$
F\left(\begin{array}{c}
\psi(H(A x, B y)), \psi(d(S x, T y)), \psi(d(S x, A x)), \\
\psi(d(T y, B y)), \psi(d(S x, B y)), \psi(d(T y, A x))
\end{array}\right) \leq 0
$$

where $\psi$ is a weak altering distance and some $F \in \mathcal{F}_{c}$.

If $(A, S)$ and $T$ satisfy $C P_{(A, S) T}$ - property, then $\mathcal{C}(B, T) \neq \emptyset$.

Moreover,

a) if $S$ is an occasionally coincidentally idempotent with respect to A, then $S$ and $A$ have a common fixed point,

b) if $T$ is an occasionally coincidentally idempotent with respect to $B$, then $T$ and $B$ have a common fixed point,

c) if the hypothesis of $a$ ) and $b$ ) hold, then $S, T, A$ and $B$ have a common fixed point.

Proof. Since $(A, S)$ and $T$ satisfy $C P_{(A, S) T}$ - property, there exists $z=$ $S u \in A u=D$ and $z \in T(X)$. Hence, there exists $v \in X$ such that $z=T v$. By (1) for $x=u$ and $y=v$ we obtain

$$
\begin{gathered}
F\left(\begin{array}{c}
\psi(H(A u, B v)), \psi(d(S u, T v)), \psi(d(S u, A u)), \\
\psi(d(T v, B v)), \psi(d(S u, B v)), \psi(d(T v, A u))
\end{array}\right) \leq 0, \\
F(\psi(H(D, B v)), 0,0, \psi(d(z, B v)), \psi(d(z, B v)), 0) \leq 0 .
\end{gathered}
$$

Since $z \in D, d(z, B v) \leq H(D, B v)$ which implies $\psi(H(z, B v)) \leq$ $\psi(H(D, B v))$. 
By $\left(F_{1}\right)$ and $(2)$ we obtain

$$
F(\psi(d(z, B v)), 0,0, \psi(d(z, B v)), \psi(d(z, B v)), 0) \leq 0
$$

a contradiction of $\left(F_{2}\right)$ if $\psi(d(z, B v))>0$. Hence $\psi(d(z, B v))=0$, which implies $d(z, B v)=0$. Hence $z=T v \in B v$ and $\mathcal{C}(B, T) \neq \emptyset$.

Moreover,

a) if $S$ is an occasionally coincidentally idempotent with respect to $A$, $z=S u=S S u=S z$ and $z$ is a fixed point of $S$.

By (1) for $x=z$ and $y=v$ we obtain

$$
F\left(\begin{array}{c}
\psi(H(A z, B v)), \psi(d(S z, T v)), \psi(d(S z, A z)), \\
\psi(d(T v, B v)), \psi(d(S z, B v)), \psi(d(T v, A z))
\end{array}\right) \leq 0
$$

Since $d(z, A z)=d(S u, A z)=d(T v, A z) \leq \psi(H(A z, B v))$, it follows that $\psi(d(z, A z)) \leq \psi(H(A z, B v))$.

By $\left(F_{1}\right)$ and $(3)$ we obtain

$$
F(\psi(d(z, A z)), 0, \psi(d(z, A z)), 0,0, \psi(d(z, A z))) \leq 0,
$$

a contradiction of $\left(F_{3}\right)$ if $\psi(d(z, A z))>0$. Hence $\psi(d(z, A z))=0$, which implies $d(z, A z)=0$. Hence $z=S z \in A z$ and $z$ is a common fixed point of $A$ and $S$.

b) If $T$ is occasionally coincidentally idempotent with respect to $B$, then $z=T v=T T v=T z$ and $z$ is a fixed point of $T$.

By (1) for $x=u$ and $y=z$ we obtain

$$
F\left(\begin{array}{c}
\psi(H(A u, B z)), \psi(d(S u, T z)), \psi(d(S u, A u)), \\
\psi(d(T z, B z)), \psi(d(S u, B z)), \psi(d(T z, A u))
\end{array}\right) \leq 0 .
$$

Since $d(z, B z)=d(S u, B z) \leq H(A u, B z)$, it follows $\psi(d(z, B z)) \leq$ $\psi(d(A u, B z))$.

Then by $(4)$ and $\left(F_{1}\right)$ we obtain

$$
F(\psi(d(z, B z)), 0,0, \psi(d(z, B z)), \psi(d(z, B z)), 0) \leq 0,
$$

a contradiction of $\left(F_{2}\right)$ if $\psi(d(z, B z))>0$. Hence $\psi(d(z, B z))=0$, which implies $d(z, B z)=0$. Hence $z=T z \in B z$ and $z$ is a common fixed point of $T$ and $B$.

c) If the hypothesis of $a$ ) and $b$ ) hold, then $A, B, S$ and $T$ have a common fixed point $z$. 
If $\psi(t)=t$, then by Theorem 1 we obtain

Theorem 2. Let $(X, d)$ be a metric space, $A, B: X \rightarrow C B(X)$ and $S, T: X \rightarrow X$ such that for all $x, y \in X$

$$
F\left(\begin{array}{c}
H(A x, B y), d(S x, T y), d(S x, A x), \\
d(T y, B y), d(S x, B y), d(T y, A x)
\end{array}\right) \leq 0
$$

for some $F \in \mathcal{F}_{c}$.

If $(A, S)$ and $T$ satisfy $C P_{(A, S) T}$ - property, then $\mathcal{C}(B, T) \neq \emptyset$.

Moreover,

a) if $S$ is an occasionally coincidentally idempotent with respect to A, then $A$ and $S$ have a common fixed point,

b) if $T$ is an occasionally coincidentally idempotent with respect to $B$, then $B$ and $T$ have a common fixed point,

c) if the hypothesis of $a$ ) and $b$ ) hold, then $A, B, S$ and $T$ have a common fixed point.

\section{Applications}

\subsection{Coincidence and common fixed points for hybrid pair of mappings satisfying contractive condition of integral type}

In [2], Branciari established the following theorem, which opened the way to the study of fixed points for mappings satisfying a contractive condition of integral type.

Theorem $3([2])$. Let $(X, d)$ be a complete metric space, $c \in(0,1)$ and $f: X \rightarrow X$ be a mapping such that for each $x, y \in X$

$$
\int_{0}^{d(f x, f y)} h(t) d t \leq c \int_{0}^{d(x, y)} h(t) d t,
$$

where $h:[0, \infty) \rightarrow[0, \infty)$ is a Lebesgue measurable mapping which is summable (i.e. with finite integral) on each compact subset of $[0, \infty)$ such that for $\varepsilon>0, \int_{0}^{\varepsilon} h(t) d t>0$.

Then, $f$ has a unique fixed point $z \in X$ such that for all $x \in X, z=$ $\lim _{n \rightarrow \infty} f^{n} x$.

Some fixed point results for mappings satisfying contractive conditions of integral type are obtained in [16], [17] and in other papers.

Lemma 1. Let $h:[0, \infty) \rightarrow[0, \infty)$ be as in Theorem 3. Then $\psi(t)=$ $\int_{0}^{t} h(x) d x$ is a weakly altering distance.

Proof. The proof follows by Lemma 2.5 [16]. 
Theorem 4. Let $(X, d)$ be a metric space, $f, g: X \rightarrow X$ and $A, B: X \rightarrow$ $C B(X), h:[0, \infty) \rightarrow[0, \infty)$ as in Theorem 3 such that for all $x, y \in X$

$$
F\left(\begin{array}{c}
\int_{0}^{H(A x, B y)} h(t) d t, \int_{0}^{d(S x, T y)} h(t) d t, \int_{0}^{d(S x, A x)} h(t) d t, \\
\int_{0}^{d(T y, B y)} h(t) d t, \int_{0}^{d(S x, B y)} h(t) d t, \int_{0}^{d(T y, A x)} h(t) d t
\end{array}\right) \leq 0
$$

for some $F \in \mathcal{F}_{c}$.

If $(A, S)$ and $T$ satisfy $C P_{(A, S) T}$ - property, then $\mathcal{C}(B, T) \neq \emptyset$.

Moreover,

a) if $S$ is an occasionally coincidentally idempotent with respect to $A$, then $S$ and $A$ have a common fixed point,

b) if $T$ is an occasionally coincidentally idempotent with respect to $B$, then $T$ and $B$ have a common fixed point,

c) if the hypothesis of a) and b) hold, then $A, B, S$ and $T$ have a common fixed point.

Proof. Let $\psi(t)$ as in Lemma 1. Then

$$
\begin{aligned}
\psi(H(A x, B y)) & =\int_{0}^{H(A x, B y)} h(t) d t, \psi(d(S x, T y))=\int_{0}^{d(S x, T y)} h(t) d t, \\
\psi(d(S x, A x)) & =\int_{0}^{d(S x, A x)} h(t) d t, \psi(d(T y, B y))=\int_{0}^{d(T y, B y)} h(t) d t, \\
\psi(d(S x, B y)) & =\int_{0}^{d(S x, B y)} h(t) d t, \psi(d(T y, A x))=\int_{0}^{d(T y, A x)} h(t) d t .
\end{aligned}
$$

By (5) we obtain

$$
F\left(\begin{array}{c}
\psi(H(A x, B y)), \psi(d(S x, T y)), \psi(d(S x, A x)), \\
\psi(d(T y, B y)), \psi(d(S x, B y)), \psi(d(T y, A x))
\end{array}\right) \leq 0,
$$

which is inequality (1) from Theorem 1 . Theorem 4 follows from Theorem 1.

By Theorem 4 and Example 3 we obtain

Theorem 5. Let $(X, d)$ be a metric space and $h(t)$ as in Theorem 3 such that for all $x, y \in X$

$$
\begin{array}{r}
\int_{0}^{H(A x, B y)} h(t) d t \leq k \max \left\{\int_{0}^{d(S x, T y)} h(t) d t, \int_{0}^{d(S x, A x)} h(t) d t,\right. \\
\left.\left.\int_{0}^{d(T y, B y)} h(t) d t\right\}, \int_{0}^{d(S x, B y)} h(t) d t, b \int_{0}^{d(T y, A x)} h(t) d t\right\},
\end{array}
$$

where $k \in[0,1)$.

Moreover, if

a) if $S$ is an occasionally coincidentally idempotent with respect to $A$, then $S$ and $A$ have a common fixed point,

b) if $T$ is an occasionally coincidentally idempotent with respect to $B$, then $T$ and $B$ have a common fixed point,

c) if the hypothesis of $a$ ) and $b$ ) hold, then $A, B, S$ and $T$ have a common fixed point. 
Remark 2. By Theorem 4 and Examples 4 - 12 we obtain new particular results.

\subsection{Fixed points for hybrid pairs of mappings satisfying $\varphi$-contractive conditions}

As in [7], let $\Phi$ be the set of all functions $\varphi:[0, \infty) \rightarrow[0, \infty)$ such that

$$
\begin{gathered}
\left(\varphi_{1}\right): \varphi(t)<t \text { for } t>0 \\
\left(\varphi_{2}\right): \varphi(0)=0
\end{gathered}
$$

In the following, we denote by $\Phi_{c}$ the set of all nondecreasing functions $\varphi:[0, \infty) \rightarrow[0, \infty)$ satisfying conditions $\left(\varphi_{1}\right)$ and $\left(\varphi_{2}\right)$.

The following functions $F: \mathbb{R}_{+}^{6} \rightarrow \mathbb{R}_{+}$satisfy conditions $\left(F_{1}\right),\left(F_{2}\right),\left(F_{3}\right)$.

Example 13. $F\left(t_{1}, \ldots, t_{6}\right)=t_{1}-\varphi\left(\max \left\{t_{2}, t_{3}, \ldots, t_{6}\right\}\right)$.

Example 14. $F\left(t_{1}, \ldots, t_{6}\right)=t_{1}-\varphi\left(\max \left\{t_{2}, t_{3}, t_{4}, \frac{t_{5}+t_{6}}{2}\right\}\right)$.

Example 15. $F\left(t_{1}, \ldots, t_{6}\right)=t_{1}-\varphi\left(\max \left\{t_{2}, \frac{t_{3}+t_{4}}{2}, \frac{t_{5}+t_{6}}{2}\right\}\right)$.

Example 16. $F\left(t_{1}, \ldots, t_{6}\right)=t_{1}-\varphi\left(\max \left\{t_{2}, \sqrt{t_{3} t_{4}}, \sqrt{t_{5} t_{6}}, \sqrt{t_{3} t_{5}}, \sqrt{t_{4} t_{6}}\right\}\right)$.

Example 17. $F\left(t_{1}, \ldots, t_{6}\right)=t_{1}-\varphi\left(a t_{2}+\frac{b \sqrt{t_{5} t_{6}}}{1+t_{3}+t_{4}}\right)$, where $a, b \geq 0$ and $a+b \leq 1$.

Example 18. $F\left(t_{1}, \ldots, t_{6}\right)=t_{1}-\varphi\left(a t_{2}+b t_{3}+c t_{4}+d t_{5}+e t_{6}\right)$, where $a, b, c, d, e \geq 0$ and $c+d \leq 1, b+e \leq 1$.

Example 19. $F\left(t_{1}, \ldots, t_{6}\right)=t_{1}-\varphi\left(a t_{2}+b \max \left\{t_{3}, t_{4}\right\}+c \max \left\{\frac{t_{3}+t_{4}}{2}\right.\right.$, $\left.\left.\frac{t_{5}+t_{6}}{2}\right\}\right)$, where $a, b, c \geq 0$ and $a+b+c \leq 1$.

Example 20. $F\left(t_{1}, \ldots, t_{6}\right)=t_{1}-\varphi\left(a t_{2}+b \max \left\{2 t_{4}+t_{5}, 2 t_{4}+t_{6}, t_{3}+t_{5}\right.\right.$ $\left.+t_{6}\right\}$ ), where $a, b \geq 0$ and $a+b \leq 1$.

By Theorem 2 and Example 13 we obtain

Theorem 6. Let $(X, d)$ be a metric space, $A, B: X \rightarrow C B(X)$ and $S, T: X \rightarrow X$ such that for all $x, y \in X$

$$
\begin{aligned}
H(A x, B y)) \leq \varphi(\max \{d(S x, T y), d(S x, A x), \\
d(T y, B y), d(S x, B y), d(T y, A x)\})
\end{aligned}
$$


for some $\varphi \in \Phi_{c}$.

If $(A, S)$ and $T$ satisfy $C P_{(A, S) T}$ - property, then $\mathcal{C}(B, T) \neq \emptyset$.

Moreover, if

a) if $S$ is an occasionally coincidentally idempotent with respect to $A$, then $A$ and $S$ have a common fixed point,

b) if $T$ is an occasionally coincidentally idempotent with respect to $B$, then $B$ and $T$ have a common fixed point,

c) if the hypothesis of $a$ ) and $b$ ) hold, then $A, B, S$ and $T$ have a common fixed point.

Remark 3. By Theorem 2 and Examples 14 - 20 we obtain new particular results.

Acknowledgement. The authors are grateful to the referees for their valuable comments and suggestions on this paper.

\section{References}

[1] Abdou A.A., Common fixed points results for multivalued mappings with some examples, J. Nonlinear Sci. Appl., 9(2016), 787-798.

[2] Branciari A., A fixed point theorem for mappings satisfying a general contractive condition of integral type, Int. J. Math. Math. Sci., 29(9)(2002), $531-536$.

[3] Imdad M., Ahmad A., Kumar S., On nonlinear non-self hybrid contractions, Rad. Mat., 10(2)(2001), 233-244.

[4] Imdad M., Chauhan S., Soliman A.H., Ahmed M.A., Hybrid fixed point theorems in symmetric spaces via common limit range property, Demonstr. Math., 47(4)(2014), 949-962.

[5] Imdad M., Chauhan S., Kumam P., Fixed point theorems for two hybrid pairs of non-self mappings under joint common limit range property in metric spaces, J. Nonlinear Convex Anal., 16(2)(2015), 243-254.

[6] Khan M.S., Swaleh M., Kumar R., Fixed point theorems by altering distances between the points, Bull. Austral. Math. Soc., 30(1984), 1-9.

[7] Matkowski J., Fixed point theorems for mappings with a contractive iterate at a point, Proc. Amer. Math. Soc., 62(2)(1977), 344-348.

[8] Pathak H.K., Rodriguez-López R., Noncommutativity of mappings in hybrid fixed point results, Bound. Value Prob., 145(2013).

[9] Popa V., Fixed point theorems for implicit contractive mappings, Stud. Cercet. Stiint., Ser. Mat., Univ. Bacău 7(1997), 129-133.

[10] Popa V., Some fixed point theorem for compatible mappings satisfying an implicit relation, Demonstr. Math., 32(1)(1999), 157-163.

[11] PopA V., A general fixed point theorem for weakly commuting multi - valued mappings, Anal. Univ. "Dunărea de Jos" Galaţi, Ser. Mat. Fiz. Mec.Teor., Fasc. II 16(22)(1999), 19-22.

[12] Popa V., A general coincidence theorem for compatible multivalued mappings satisfying an implicit relation, Demonstr. Math., 33(1)(2000), 159-164. 
[13] PopA V., Coincidence and fixed point theorems for noncontinuous hybrid contractions, Nonlinear Anal. Forum., 7(2)(2002), 153-158.

[14] PopA V., Altering distance and strict fixed points for multifunctions satisfying implicit relation, Bul. Inst. Politehn. Iaşi, Ser. Mat. Mec. Teor. Fiz., 57(61)(2)(2011), 1-9.

[15] Popa V., A general coincidence and common fixed theorem for two hybrid pairs of mappings, Demonstr. Math., 47(4)(2014), 971-978.

[16] Popa V., Mocanu M., Altering distance and common fixed points under implicit relations, Hacet. J. Math. Stat., 38(3)(2009), 329-337.

[17] Rhondes B.E., Two fixed point theorems for mappings satisfying a general contractive condition of integral type, Int. J. Math. Math. Sci., 63(2003), 4007-4013.

[18] Sastry K.P.R., Babu G.V.R., Fixed point theorems in metric spaces by altering distances, Bull. Calcutta Math. Soc., 90(3)(1998), 175-182.

[19] Sastry K.P.R., Babu G.V.R., Some fixed point theorems by altering distances between the points, Indian J. Pure Appl. Math., 30(6)(1999), 641-647.

[20] Sintunavarat W., Kumam P., Common fixed point theorems for a pair of weakly compatible mappings in fuzzy metric spaces, Appl. Math. 2011(2011), Article ID 637958, 14 pages.

\author{
VALERIU POPA \\ "Vasile Alecsandri" University of BacĂU \\ 157 CALEA MĂRĂŞEŞTI \\ BACĂU, 600115, ROMANIA \\ e-mail: vpopa@ub.ro \\ Alina-Mihaela Patriciu \\ DunăRea de Jos" University of Galaţi \\ FACUlty of SCIENCES AND EnVIRONMENT \\ Department of Mathematics and Computer Sciences \\ 111 Domnească Street \\ Galaţi, 800201, Romania \\ e-mail: Alina.Patriciu@ugal.ro
}

Received on 24.11.2016 and, in revised form, on 28.04.2017. 